\title{
Etiology and Pathophysiology of Diverticular Disease
}

\author{
Kristina G. Hobson, M.D. ${ }^{1}$ and Patricia L. Roberts, M.D. ${ }^{1}$
}

\begin{abstract}
Diverticular disease is prevalent in Western countries and yet its pathogenesis remains poorly understood. The common theories of the cause of diverticular disease have focused on colonic motility, the structural aspects of the colonic wall, and the role of dietary factors, particularly fiber.
\end{abstract}

KEYWORDS: Diverticular disease, segmentation, elastosis, colonic motility

Objectives: On completion of this article, the reader should be able to summarize the pathophysiology of the development of diverticular disease.

Diverticular disease is largely a disease of the twentieth century. While diverticula were described by Littre in the late 1700 s, ${ }^{1}$ it was Graser in $1899^{2}$ who actually described the condition of diverticulitis and Beer in $1904^{3}$ who correlated the histologic and clinical features.

Colonic diverticulosis is common in industrialized nations and the prevalence increases dramatically with increasing age. Under the age of 30 , about 1 to $2 \%$ of patients will have diverticulosis, while diverticulosis is found in over $40 \%$ of the population after age $60 .{ }^{4}$ Approximately 10 to $25 \%$ of patients with diverticulosis will develop diverticulitis with a range of symptoms from mild abdominal pain to frank fecal peritonitis. Studies of Western populations demonstrate a tendency for diverticulosis to occur on the left side, primarily in the sigmoid colon (55 to $95 \%) .{ }^{5-8}$ With increasing age, the total number of diverticula increases, and they occur progressively more proximally. As a result, right-sided diverticular disease in Western societies is observed primarily in older patients in the setting of pan-diverticulosis with contiguous proximal spread. ${ }^{5,6}$ In contrast, diverticular disease in Asian countries occurs much more commonly on the right, with as many as $70 \%$ of the diverticula isolated to the right side. ${ }^{9-11}$

While the terms diverticulosis, diverticulitis, and diverticular disease are often used interchangeably, diverticulosis refers to the presence of diverticula in the colon, diverticulitis refers to the presence of peridiverticular inflammation and infection, and diverticular disease refers to the full spectrum of symptoms caused by the presence of diverticula including cramping, bloating, pain, and fever.

\section{PATHOGENESIS}

The common theories with respect to the development of diverticulosis focus on three areas: structural abnormalities of the colonic wall, disordered motility, and the role of dietary fiber.

Diverticular Disease; Editor in Chief, David E. Beck, M.D.; Guest Editor, Richard E. Karulf, M.D. Clinics in Colon and Rectal Surgery, volume 17, number 3, 2004. Address for correspondence and reprint requests: Patricia L. Roberts, M.D., Department of Colon and Rectal Surgery, Lahey Clinic, 41 Mall Rd., Burlington, MA 01805. E-mail: Patricia.L.Roberts@Lahey.org. ${ }^{1}$ Department of Colon and Rectal Surgery, Lahey Clinic, Burlington, Massachusetts. Copyright (C) 2004 by Thieme Medical Publishers, Inc., 333 Seventh Avenue, New York, NY 10001, USA. Tel: +1(212) 584-4662. 1531-0043,p;2004,17,03,147,153,ftx,en;ccrs00177x. 


\section{Colonic Wall Resistance/Structural Aspects}

The normal colonic wall consists of mucosa, submucosa, and muscle layers. The muscularis propria consists of an inner layer of circular muscle and an outer layer that is concentrated mainly in three narrow bands known as taeniae coli. Two of the taeniae in the sigmoid colon are located on the antimesenteric border of the colon and the third is located on the mesenteric border. The muscle layer becomes progressively thicker in the more distal colon, particularly the sigmoid colon, and the taeniae eventually fuse in the proximal rectum, a landmark useful for identifying the distal extent of resection when performing a sigmoid colectomy.

Diverticula occur in areas of relative colonic wall weakness passing through the circular muscle layer where blood vessels (vasa recta) penetrate to supply the mucosa. They generally occur in parallel rows along the mesenteric side of the antimesenteric taeniae; with progression, an additional row of diverticula may be found between the antimesenteric taeniae. ${ }^{12}$ Microscopic studies of areas of the colon with early small diverticula demonstrate areas of thinning due to presumed focal microscopic muscle atrophy. ${ }^{5}$ With progression, a clear-cut defect in the muscle occurs, usually at the site of penetration of a vessel though gaps in the circular muscle layer. ${ }^{5}$ Interestingly, diverticula remain isolated to the circular muscle, and rarely, if ever, penetrate through the taeniae. ${ }^{12}$

The reason for this predilection for development of diverticula along the mesenteric side of the antimesenteric taeniae of the colon is unclear. The most likely explanation relates to the colonic muscular blood supply which arises from the mesenteric side of the colon. Large circumferential vessels course over the top of diverticula, with small perforators passing along the neck of the diverticulum toward the bowel lumen. ${ }^{12,13}$ These perforating mucosal vessels create a weak point in the circular muscle coat that is susceptible to herniation. As the perforating vessels on the mesenteric side are more proximal branches and are of larger caliber, these vessels presumably create a larger potential weak point, thus explaining the progression of diverticula from the mesenteric to the antimesenteric side only after years of progressive disease.

Striking changes are seen in both the circular muscle layer and taeniae in patients with diverticular disease. ${ }^{14}$ Early necropsy studies describe increased bowel wall thickness in patients with diverticular disease $^{12}$ and although this was initially felt to be from muscular hypertrophy, multiple subsequent studies have shown that there is no evidence of either hypertrophy or hyperplasia. Whiteway and Morson studied the muscularis propria in patients with uncomplicated diverticulosis and found that the taenia was thickened secondary to elastin deposition. ${ }^{15}$ There was an increase of over 200\% in elastin in these patients compared with controls. As the elastin was laid down in a contracted form, shortening of the taeniae led to a bunching of the circular muscle, leading to the accordion-like or so-called concertina-like effect on the bowel. Thus, the remarkable muscular thickening found in association with diverticular disease represented an exaggerated contraction of normal colonic myocytes. ${ }^{14}$ Furthermore, subsequent studies have shown that muscular thickening extends only two thirds of the way around the circumference of the bowel, between the mesenteric and antimesenteric taeniae. $^{12,16}$ This anatomical isolation of the muscle spasm corresponds with the areas of heaviest concentration of diverticula, as described earlier.

Despite the grossly increased muscle wall thickness, the colon wall in patients with diverticulosis is more susceptible to herniation. The role of collagen and the tensile strength of the colonic wall have been investigated. Wess et $\mathrm{al}^{17}$ analyzed colonic collagen content in an attempt to determine if a lack of collagen is responsible for this apparent weakness of the muscular wall. As the collagen content does not change with age or the presence of diverticula, the changes are more likely to be qualitative than quantitative. Collagen fibrils demonstrate increased cross-linking with increased age; this process seems to increase most dramatically after 40 years of age, the age at which the incidence of diverticular disease also appears to increase. This same study demonstrated that patients with diverticulosis have an abnormally high amount of collagen cross-linkage in the colon wall. This difference persisted even when patients were compared with age-matched controls. This increased cross-linkage likely causes the tissues to become stiffer and less resistant to stretching. This loss of compliance of the colonic submucosa, the layer primarily responsible for tensile strength, may make the submucosa more susceptible to small tears when subjected to the higher intraluminal pressures triggered by segmentation. Any tear in this strength layer could potentially then lead to mucosal herniation and the premature formation of diverticular disease. A possible genetic connective tissue defect has been suggested because of reports of diverticula in young patients with Marfan syndrome or Ehlers-Danlos syndrome. ${ }^{18,19}$

\section{Colonic Motility}

In addition to the structural changes in the colonic wall, alterations in colonic motility have been implicated in the development of diverticular disease.

Painter and colleagues performed sigmoid manometry and cineradiography in patients with and without diverticular disease. ${ }^{20}$ They found that fecal material normally passes through the length of the colon with minimal change in pressure. However, when two haustral contractions occur in a given segment of the colon at the same time, it can cause temporary isolation of that 
segment of bowel, a process called segmentation. This process may be likened to the development of small compartments in the sigmoid colon or "small bladders." Thus, the colon seemed to function not as a tube, but as individual compartments generating high pressures. Contraction of the bowel wall subsequently causes locally increased intracolonic pressure, resulting in a functional obstruction. In this way, segmentation creates a pulsion force causing visible distension of the local diverticula. ${ }^{14}$ The high pressures resulting from segmentation in turn lead to the focal muscular atrophy and subsequent mucosal herniation. ${ }^{10}$ Pulsion diverticula occur most frequently in the sigmoid colon because the lumen of the colon is the narrowest resulting in the generation of the highest pressures.

Painter showed that the normal sigmoid colon had a basal pressure of a few millimeters of mercury above atmospheric pressure and the basal pressure was the same in patients with and without diverticular disease. $^{21}$ High pressure waves also occur, which are independent of peristaltic waves. In normal subjects these waves have an amplitude of $10 \mathrm{~mm} \mathrm{Hg}$; however, in patients with diverticular disease, the amplitude of such waves may be as high as $90 \mathrm{~mm} \mathrm{Hg}$ and last for 10 to 20 seconds. Segments of the colon also generate high pressure waves in response to administration of morphine sulfate and prostigmine but not to meperedine.

Studies investigating intraluminal pressures in the colon and the relationship to the development of diverticular disease have multiple drawbacks. As pointed out by Parks and Connell, ${ }^{22}$ there are methodological differences particularly related to bowel preparation and to the various pressure sensors used. Some studies have measured a relatively short time period (i.e., 2 hours, although time periods of up to 24 hours may be necessary to draw valid conclusions) for study and small numbers of patients in other studies have limited meaningful conclusions.

Other investigators have focused on myoelectrical activity in the colon to explain the development of diverticulosis. Myoelectrical studies have shown distinct slow wave motility patterns in patients with diverticular disease compared with irritable bowel syndrome. ${ }^{23}$ Patients with diverticular disease have abnormal slow wave motility patterns of 12 to 18 cycles per minute, while patients with irritable bowel syndrome have a 3cycle-per-minute motility pattern. ${ }^{23,24}$ Administration of bran has no effect on the motility pattern in patients with irritable bowel syndrome ${ }^{25}$; however, administration of bran in patients with diverticular disease causes return of the motility pattern to normal. Of interest, some of these strong contractions persisted in patients despite sigmoid resection. ${ }^{26}$

In addition to the development of high intraluminal pressures, several studies have also demonstrated increased colonic motility in the setting of diverticular disease. $^{27,28}$ The hypermotility appears to predominate in the descending and sigmoid colon, while motility is normal in the transverse colon. ${ }^{28}$ The motility is also disorganized, leading to frequent retrograde propagation of propulsions. ${ }^{28}$ The etiology of this uncoordinated and excessive activity is unclear. An isolated study of colonic electrical activity in diverticular disease demonstrated no defect in normal slow wave frequency compared with normal controls. ${ }^{29}$ Thus an underlying baseline electrical activity does not appear to be responsible for the hyperactivity seen in diverticular disease.

The role of chemical mediators on colonic motility has also been evaluated. Numerous chemical mediators of bowel motility are found within the nerve cell bodies of the colon. A study of colonic vasoactive intestinal polypeptide (VIP) levels in patients with diverticular disease demonstrated significantly higher levels of VIP in the mucosa and nonmuscular layers of the bowel wall compared with normal colons, while the levels in the circular muscle and taeniae coli were normal. ${ }^{30}$ Neuropeptide $\mathrm{Y}$ and substance $\mathrm{P}$ levels were also measured in the colonic wall and were normal in patients with diverticulosis. Although muscular levels of VIP appear to be normal, the increased total wall content may still contribute to the intracolonic high pressures seen in earlier studies by Painter. An extensive array of chemical mediators of bowel motility exists that has not yet been evaluated in the setting of diverticular disease. Future studies of these various mediators have great potential for improving our understanding of the pathophysiology of diverticular disease.

\section{Dietary Factors and the Role of Fiber}

Diverticular disease has been called a disease of Western civilization and a large body of evidence supports the role of diet, particularly fiber, in the pathogenesis of the disease.

Authors of early studies of diverticular disease primarily made their conclusions by comparing populations with traditionally low incidence of diverticular disease (such as Asian or African populations) with Westernized populations that have an ever-increasing incidence of diverticular disease. A striking difference between these two populations is the diet. Painter and Burkitt $^{31}$ noted that the most striking difference in the diets of the two populations was the amount of dietary fiber consumed. They studied colonic transit times and stool weights in over 1,000 individuals in the United Kingdom and sub-Saharan Africa. Longer transit times and lower stool weights were seen in the UK population than in the Ugandan population. A high-fiber diet was felt to be the major contributing factor to faster colonic transit times, larger stool volumes, and more frequent bowel movements. They reasoned that the rising incidence of diverticular disease in the Western world could 
potentially be due to a gradual decrease in consumption of dietary fiber over the course of the last century. Specifically, the advent of roller milling and the process of refining sugar during the Industrial Revolution removed a large source of fiber from Western diets. This would explain why the first reports of increasing diverticular disease appear in the literature about the time the first cohort of children raised on refined sugar and white flour came of the age where diverticular disease begins to occur, about 40 years after the start of the Industrial Revolution. Mendeloff ${ }^{32}$ criticized this theory as oversimplified and felt the differences between sub-Saharan Africa and Western cultures were too great to conclude that a lack of fiber was the only difference responsible for the increased incidence of diverticular disease.

Animal studies have supported the role of dietary fiber in the development of diverticular disease. In a prospective randomized trial of the effects of differing amounts of dietary fiber in rats, Fisher and colleagues demonstrated a dramatic increase in the number of rats with diverticulosis when fed low-fiber diets compared with those fed high-fiber diets. ${ }^{33}$ In this study, 1800 rats were fed diets of varying fiber content. Approximately $45 \%$ of the rats on the lowest fiber diet developed diverticula compared with $9 \%$ of the rates on the highest fiber diet. The rat colon is different from the human colon in that the longitudinal layer surrounds the colon; in addition the rats developed diverticula on the right side of the colon, at points of blood vessel penetration similar to the human diverticula.

This debate has stimulated several studies on the effects of a high-fiber diet on the colon. Findlay and associates ${ }^{34}$ found improved intestinal transit time after only a 1-month course of high-fiber diet in patients with diverticular disease. However, stool weight did not change significantly. In contrast, Taylor and Duthie ${ }^{23}$ did find increased stool weight, decreased transit time, and decreased motility, as well as symptomatic improvement in patients with diverticular disease after supplementing their diets with bran tablets.

Manousos ${ }^{35}$ and Aldoori ${ }^{36}$ and their respective coworkers individually examined dietary factors in large populations of patients both with and without diverticular disease. Both studies demonstrated significantly lower consumption of dietary fiber in patients with diverticular disease when compared with their healthy counterparts. They also found that patients with diverticulosis also tended to eat greater quantities of red meat and fats. Aldoori studied 51,529 male health professionals prospectively, and over a 6-year period there were $384(0.75 \%)$ new cases of diverticular disease. The risk of the development of diverticular disease was inversely associated with insoluble dietary fiber intake. Fiber found in fruits and vegetables conferred the most protective effect (compared with fiber from cereal) and a high intake of total fat and red meat increased the incidence of diverticular disease. Although these studies have potential confounding variables including detection and recall bias, they support the role of dietary fiber. Due to the cost and the large numbers of patients that would have to be accrued, it is unlikely that a prospective, randomized, controlled trial on the effect of dietary fiber intake on the development of colonic diverticula would be able to be accomplished.

\section{Additional Factors}

Several additional factors may contribute to the development of diverticular disease.

Physical activity. Two studies have looked at the effect of exercise on diverticular disease. Aldoori and colleagues ${ }^{36}$ evaluated the characteristics of 382 men in the health professionals cohort who developed diverticular disease over a 4-year period and compared them to those of the almost 48,000 men without symptomatic diverticular disease. The amount of physical activity and the risk of developing symptomatic diverticular disease were inversely related. This difference persisted even when any differences in dietary fiber intake were controlled for. A potential drawback of the study is that the differences may have arisen merely from the fact that exercise might have been impaired or prohibited by the symptoms of diverticular disease. ${ }^{37}$

Smoking. The Health Professionals' Consortium Study was unable to demonstrate any relationship between the use of tobacco products and the development of diverticular disease. ${ }^{38}$ However, once patients develop diverticular disease, evidence suggests smoking may make them more susceptible to complications. In one study, $53 \%$ of all patients with complicated diverticular disease smoked, compared with only $29 \%$ of the patients with uncomplicated disease. ${ }^{39}$

Nonsteroidal anti-inflammatory medications. Nonsteroidal anti-inflammatory medications (NSAIDs) have been associated with multiple gastrointestinal complications, such as the exacerbation of gastric ulcers, and hemorrhage. Evidence suggests chronic NSAID use is almost twice as common in patients with diverticular disease as it is in healthy controls with no known colonic disease. $^{40,41}$ The health professionals follow-up cohort also documented an increased incidence of uncomplicated but symptomatic diverticular disease in patients who used NSAIDs regularly compared with their asymptomatic counterparts. ${ }^{42}$ NSAID use may also increase the risk of complications of diverticulitis. A retrospective study by Corder documented a $23 \%$ higher risk of perforating diverticulitis in patients who took NSAIDs regularly compared with patients with diverticular disease who did not take NSAIDs. ${ }^{43}$ A subsequent study by Wilson and colleagues ${ }^{44}$ found the incidence of chronic NSAID use among patients admitted to the hospital 
for diverticular disease was significantly higher than in the population as a whole. Furthermore, these patients were more than four times as likely to perforate from their disease than patients with no history of NSAID use.

Caffeine ingestion. Caffeine intake has also been investigated as a possible contributing factor to the development of diverticular disease. Since caffeine stimulates small bowel secretion and may also affect colonic transit time, ${ }^{45}$ a possible relationship, either causative or preventative, between caffeine intake and the risk of diverticular disease has been postulated. However, when caffeine consumption was evaluated in patients both with and without symptomatic diverticular disease, no difference between the two groups was found. ${ }^{38}$

\section{DEVELOPMENT OF SYMPTOMS}

Although the majority of patients with diverticula remain asymptomatic, approximately 10 to $25 \%$ of patients develop symptoms, which may range from significant abdominal pain associated with fever and leukocytosis requiring hospitalization to a large proportion of patients with recurrent, short-lived abdominal pain which may have a significant impact on lifestyle and be associated with alteration in bowel movements. ${ }^{46}$ Why some patients with diverticulosis remain asymptomatic while others develop symptoms is unknown, but it has been suggested that the development of symptoms involves several inter-related processes including muscular dysfunction, inflammation and visceral hypersensitivity. ${ }^{47}$

\section{Inflammation}

Inflammatory change associated with diverticulitis is most often termed "acute diverticulitis," describing systemic symptoms associated with peritoneal inflammation including left lower quadrant pain, fever, and leukocytosis. However, it is also recognized that luminal mucosal inflammation may be associated with colonic diverticula and have some features of inflammatory bowel disease. ${ }^{48}$ This entity has been called segmental colitis, sigmoiditis, and diverticular colitis. ${ }^{4-51}$ The clinical significance of this type of diverticula-associated inflammation or colitis is unknown; the incidence is low, found in about 0.25 to $1.5 \%$ of colonoscopies performed. ${ }^{48,52}$

Similarly, the degree of inflammation has been difficult to correlate with symptoms. Horgan and colleagues retrospectively reviewed 47 patients who underwent sigmoid resection for diverticular disease. ${ }^{53}$ They reported a syndrome of "smoldering diverticulitis" that occurred in patients with chronic left lower quadrant pain but no associated fever or leukocytosis. Seven patients had previously undetected pericolic abscesses and $76 \%$ had evidence of acute and chronic mucosal inflammation (while only 13\% had it detected preoperatively). Over three quarters of patients had resolution of symptoms after resection. There was no correlation between the extent of resection, the type of duration of symptoms, and the preoperative endoscopic evidence of inflammation. Similarly, Morson ${ }^{14}$ found no evidence of colonic inflammation in one third of patients who had a resection for diverticular disease. While some patients may have resolved the inflammatory change prior to elective resection, this suggests again a weak correlation, at best between the degree of inflammation and symptoms in diverticular disease.

\section{Visceral Hypersensitivity}

Persistent colonic symptoms, particularly abdominal pain, have been reported after resolution of infectious enteritis and inflammatory bowel disease and may also occur after an episode of acute diverticulitis. It has been suggested that this sharp pain represents heightened visceral sensitivity. ${ }^{47}$ Visceral hypersensitivity has been used to describe excessive perception or an excessive afferent response to stimuli. While the majority of studies have been done in irritable bowel syndrome, there is a suggestion that this sensitivity may contribute to symptoms in diverticular disease. Furthermore, patients with acute diverticulitis have increased nerve staining in the muscularis propria compared with control patients, in addition to increased staining in the mucosa and submucosa. ${ }^{54}$ Also, the neurons are of smaller diameter suggesting proliferation in response to inflammation. Nerve damage in patients who have had diverticulitis has been documented and it is postulated that this may represent regeneration and hyperinnervation and may contribute to symptoms. ${ }^{54-56}$

While earlier theories have examined the role of fiber and colonic pressures in the pathogenesis of diverticular disease, correlation with precise symptomatology has been difficult. Exploring and investigating additional entities, such as the relationship between inflammation, visceral sensitivity, and enteric peptides, is likely to further our understanding of diverticular disease and, it is hoped, guide diagnosis and treatment.

\section{REFERENCES}

1. Finney JMT. Diverticulitis and its surgical treatment. Proc Interstate Post-Grad Med Assembly North Am 1928;55: 57-65

2. Graser E. Das falsche Darmdivertikel. Arch klin Chir 1899; 59:638-647

3. Beer E. Some pathological and clinical aspects of acquired (false) diverticula of the intestine. Am J Med Sci 1904;128: 135-145

4. Parks TG. Natural history of diverticular disease of the colon: a review of 521 cases. BMJ 1969;4:639-645 
5. Hughes LE. Postmortem survey of diverticular disease of the colon. II. The muscular abnormality of the sigmoid colon. Gut 1969;10:344-351

6. Boles RS Jr, Jordan SM. The clinical significance of diverticulosis. Gastroenterology 1958;35:579-582

7. Zollinger RW. The prognosis in diverticulitis of the colon. Arch Surg 1968;97:418-422

8. Rodkey GV, Welch CE. Changing patterns in the surgical treatment of diverticular disease. Ann Surg 1984;200:466-478

9. Nakada I, Ubukata H, Goto Y, et al. Diverticular disease of the colon at a regional general hospital in Japan. Dis Colon Rectum 1995;38:755-759

10. Chia JG, Wilde CC, Ngoi SS, Goh PM, Ong CL. Trends of diverticular disease of the large bowel in a newly developed country. Dis Colon Rectum 1991;34:498-501

11. Sugihara K, Muto T, Morioka Y, Asano A, Yamamoto T. Diverticular disease of the colon in Japan. A review of 615 cases. Dis Colon Rectum 1984;27:531-537

12. Slack WW. The anatomy, pathology and some clinical features of diverticulitis of the colon. Br J Surg 1962;50: 185-190

13. Meyers MA, Volberg F, Katzen B, Alonso D, Abbott G. The angioarchitecture of colonic diverticula. Significance in bleeding diverticulosis. Radiology 1973;108:249-261

14. Morson BC. The muscle abnormality in diverticular disease of the colon. Proc R Soc Med 1963;56:798-800

15. Whiteway J, Morson BC. Elastosis in diverticular disease of the sigmoid colon. Gut 1985;26:258-266

16. Hughes LE. Postmortem survey of diverticular disease of the colon. I. Diverticulosis and diverticulitis. Gut 1969;10:336344

17. Wess L, Eastwood MA, Wess TJ, Busuttil A, Miller A. Cross-linking of collagen is increased in colonic diverticulosis. Gut 1995;37:91-94

18. Cook JM. Spontaneous perforation of the colon: report of two cases in a family exhibiting Marfan's stigmata. Ohio State Med J 1968;64:73

19. Beighton PH, Murdoch JL, Votteler T. Gastrointestinal complications of the Ehlers-Danlos syndrome. Gut 1969;10: 1004-1008

20. Painter NS, Truelove SC, Ardran GM, Tuckey M. Segmentation and the localization of intraluminal pressures in the human colon, with special reference to the pathogenesis of colonic diverticula. Gastroenterology 1965;49:169-177

21. Painter NA. The aetiology of diverticulosis of the colon with special reference to the action of certain drugs on the behaviour of the colon. Ann R Coll Surg Engl 1964;34:98119

22. Parks TG, Connell AM. Motility studies in diverticular disease of the colon. Gut 1969;10:534-542

23. Taylor I, Duthie HL. Bran tablets and diverticular disease. BMJ 1976;1:988-990

24. Snape WJ Jr, Carlson GM, Cohen S. Colonic myoelectrical activity in the irritable bowel syndrome. Gastroenterology 1976;70:326-330

25. Taylor I, Darby C, Hammond P. Comparisons of rectosigmoid myoelectrical activity in the irritable colon syndrome during relapses and remissions. Gut 1978;19:923-929

26. Parks TG. Rectal and colonic studies after resection of the sigmoid for diverticular disease. Gut 1970;11:121-125

27. Trotman IF, Misiewicz JJ. Sigmoid motility in diverticular disease and the irritable bowel syndrome. Gut 1988;29:218222
28. Bassotti G, Battaglia E, Spinozzi F, Pelli MA, Tonini M. Twenty-four-hour recordings of colonic motility in patients with diverticular disease: evidence for abnormal motility and propulsive activity. Dis Colon Rectum 2001;44:18141820

29. Katschinski M, Lederer P, Ellermann A, Ganzleben R, Lux G, Arnold R. Myoelectric and manometric patterns of human rectosigmoid colon in irritable bowel syndrome and diverticulosis. Scand J Gastroenterol 1990;25:761-768

30. Milner P, Crowe R, Kamm MA, Lennard-Jones JE, Burnstock G. Vasoactive intestinal polypeptide levels in sigmoid colon in idiopathic constipation and diverticular disease. Gastroenterology 1990;99:666-675

31. Painter NA, Burkitt DP. Diverticular disease of the colon: a deficiency disease of Western civilization. BMJ 1971;2:450 454

32. Mendeloff AI. A critique of "fiber deficiency." Am J Dig Dis 1976;21:109-112

33. Fisher N, Berry CS, Fearn T, Gregory JA, Hardy J. Cereal dietary fiber consumption and diverticular disease: a lifespan study in rats. Am J Clin Nutr 1985;42:788-804

34. Findlay JM, Smith AN, Mitchell WD, Anderson AJ, Eastwood MA. Effects of unprocessed bran on colon function in normal subjects and in diverticular disease. Lancet 1974; 1:146-149

35. Manousos O, Day NE, Tzonou A. Diet and other factors in the aetiology of diverticulosis: an epidemiological study in Greece. Gut 1985;26:544-549

36. Aldoori WH, Giovannucci EL, Rimm EB, Wing AL, Trichopoulos DV, Willett WC. A prospective study of diet and the risk of symptomatic diverticular disease in men. Am J Clin Nutr 1994;60:757-764

37. Heaton KW, Thompson WG. Exercise and diverticular disease. BMJ 1995;310:1332

38. Aldoori WH, Giovannuci EL, Rimm EB, Wing AL, Trichopoulos DV, Willett WC. A prospective study of alcohol, smoking, caffeine and the risk of symptomatic diverticular disease in men. Ann Epidemiol 1995;5:221228

39. Papagrigoriadis S, Macey L, Bourantas N, Rennie JA. Smoking may be associated with complications in diverticular disease. Br J Surg 1999;86:923-926

40. Campbell K, Steele RJ. Non-steroidal anti-inflammatory drugs and complicated diverticular disease: a case-control study. Br J Surg 1991;78:190-191

41. Goh H, Bourne R. Non-steroidal anti-inflammatory drugs and perforated diverticular disease: a case-control study. Ann R Coll Surg Engl 2002;84:93-96

42. Aldoori WH, Giovannucci EL, Rimm EB, Wing AL, Willett WC. Use of acetaminophen and nonsteroidal anti-inflammatory drugs: a prospective study of the risk of symptomatic diverticular disease in men. Arch Fam Med 1998;7:255260

43. Corder A. Steroids, non-steroidal anti-inflammatory drugs, and serious septic complications of diverticular disease. $\mathrm{Br}$ Med J (Clin Res Ed) 1987;295:1238

44. Wilson RG, Smith AN, Macintyre IM. Complications of diverticular disease and non-steroidal anti-inflammatory drugs: a prospective study. Br J Surg 1990;77:1103-1104

45. Wald A, Back C, Bayless TM. Effect of caffeine on the human small intestine. Gastroenterology 1976;71:738-742

46. Simpson J, Spiller RC. Colonic diverticular disease. Clin Evid 2002;7:398-405 
47. Simpson J, Scholefield JH, Spiller RC. Origin of symptoms in diverticular disease. Br J Surg 2003;90:899-908

48. Shepherd NA. Diverticular disease and chronic idiopathic inflammatory bowel disease: associations and masquerades. Gut 1996;38:801-802

49. Makapugay LM, Dean PJ. Diverticular disease-associated chronic colitis. Am J Surg Pathol 1996;20:94-102

50. Gledhill A, Dixon MF. Crohn's-like reaction in diverticular disease. Gut 1998;42:392-395

51. Gore S, Shepherd NA, Wilkinson SP. Endoscopic crescentic fold disease of the sigmoid colon: the clinical and histopathological spectrum of a distinctive endoscopic appearance. Int J Colorectal Dis 1992;7:76-81

52. Imperiali G, Meucci G, Alvisi C, et al. Segmental colitis associated with diverticula: a prospective study. Gruppo di
Studio per le Mallattie Infiammatorie Intestinali (GSMII). Am J Gastroenterol 2000;95:1014-1016

53. Horgan AF, McConnell EJ, Wolff BG, The S, Paterson C. Atypical diverticular disease: surgical results. Dis Colon Rectum 2001;44:1315-1318

54. Simpson J, Haji-Suyoi A, Jenkins D, Scholefield JH, Spiller RC. Quantification of neurological changes in resection specimens with complicated and uncomplicated diverticular disease. Gastroenterology 2002;122:A314

55. Stead RH. Nerve remodelling during intestinal inflammation. Ann N Y Acad Sci 1992;664:443-455

56. Brewer DB, Thompson H, Haynes IG, Alexander-Williams J. Axonal damage in Crohn's disease is frequent, but nonspecific. J Pathol 1990;161:301-311 